\title{
Intermediate-age globular clusters in merger remnants
}

\author{
Paul Goudfrooij \\ Space Telescope Science Institute, 3700 San Martin Drive, Baltimore, MD 21218, USA
}

\begin{abstract}
Recent observations of globular clusters in intermediate-age (2-4 Gyr old) merger remnants have provided the hitherto "missing link" between between young merger remnants and normal elliptical galaxies. The luminosity functions (LFs) of the "blue" globular clusters exhibit a Gaussian shape centered on $M_{V} \sim-7.2$ as found for giant ellipticals, while the "red" clusters exhibit LFs that are more extended towards both the bright and faint ends, as expected for a cluster population of intermediate age. Specific frequencies for these systems have been calculated by evolving both the LF of the "red" clusters and the parent galaxy luminosity to an age of 10 Gyr. They are found to be consistent with the values found for giant ellipticals in poor clusters. These findings support the viability of the "merger scenario" for forming the "red" GCs in giant early-type galaxies.
\end{abstract}

\section{Introduction}

This workshop did a great job in re-emphasizing that the study of globular clusters (GCs) around galaxies provides relevant and unique information regarding our understanding of the formation history of galaxies. Their nature as simple stellar population significantly simplifies the determination of their ages and metallicities relative to that of stellar populations that constitute the integrated light of their parent galaxies.

A well-known, interesting feature of the GC systems of giant early-type galaxies is the presence of bimodal color distributions, providing obvious evidence for the occurrence of a 'second event' during the formation history of these galaxies. To date, three main competing concepts to explain this phenomenon have been proposed in the literature. The first one (and the only one to have actually predicted this bimodality) is the "merger scenario" by Ashman \& Zepf [1], who modeled the properties of GCs forming in a major merger of gas-rich spirals (together with the giant elliptical host galaxies themselves). They figured that the pre-enriched gas associated with the spiral disks would form 'red' (i.e., metalrich) GCs during the merger. The two other popular concepts are the "accretion scenario" [2] and the "multi-phase collapse scenario" [3], both of which do not involve new GCs formed in a merger. More details on these three scenarios (incl. their pros and cons) are discussed in A. Kundu's contribution to this volume.

Given these rather different points of view, it is important to review the direct evidence presented by observations of merger remnants to test the predictions of the "merger scenario" for forming the red GCs in giant early-type galaxies. This is the main purpose of the remainder of this paper. 
Until just a year or so ago, strong evidence for luminous GCs having formed during major, gas-rich mergers was limited to a half dozen cases, namely 2 ongoing mergers (NGC 4038/4039 [4], NGC 3256 [5], and 4 young merger remnants (NGC 1275 [6], NGC 3921 [7], NGC 3597 [8], NGC 7252 [9]). These authors determined ages of the luminous GCs in these galaxies from photometric and/or spectroscopic data. Their ages are typically $\lesssim 500 \mathrm{Myr}$, a time at which most GC destruction and evolution processes have only just started.

It is therefore perhaps not surprising that major sources of debate in the context of the nature of the red GCs in giant ellipticals are: (i) whether or not the GCs formed in mergers will reach old age, (ii) whether or not the metallicities of GCs formed in mergers are compatible with those of 'red' GCs in old ellipticals, and (iii) whether or not the luminosity functions and specific frequencies of GCs formed in mergers will become compatible with those of 'red' GCs in old ellipticals at the latter's age. We discuss these items below along with very recent results on the GC systems of more evolved merger remnants.

\section{$2 \quad$ The IMF and Metallicity of GCs Formed in Mergers}

One important issue in figuring out whether luminous GCs can survive to old age is the shape of the initial mass function (IMF) of the (individual) GCs. Brodie et al. [6] obtained spectra of luminous GCs in NGC 1275 and found $\mathrm{H} \gamma$ and $\mathrm{H} \delta$ equivalent widths that are somewhat larger (and $B-R$ colors that are somewhat bluer) than those of the 1995 version of the Bruzual \& Charlot population synthesis models in the appropriate age range, both for Salpeter or Scalo IMFs. They showed that the large Balmer line equivalent widths could only be brought into agreement with those models by assuming a truncated IMF, e.g., involving a mass range of $2-3 \mathrm{M}_{\odot}$. For such a high low-mass cutoff, they argue that these clusters will fade away in only $\sim 1$ Gyr. On the other hand, Schweizer \& Seitzer [10] showed that similarly old ( 500 Myr) GCs in NGC 7252 show Balmer line strengths that are well reproduced by the Bruzual \& Charlot (1996; BC96) models (which indeed produce larger Balmer equivalent widths than their 1995 models). Also, Gallagher \& Smith [11] found no need to invoke a truncated IMF to explain the Balmer line strengths in young clusters in the starburst galaxy M 82. But then again, a detailed study of the very luminous star cluster M 82-F [12 revealed that a lower mass cutoff of $2-3 \mathrm{M}_{\odot}$ is required (for a Salpeter IMF) to match their observations. Obviously, the IMF among luminous young star clusters may not be universal.

Another open question until a year or so ago was whether or not the metallicities of GCs formed in mergers are compatible with those of 'red' GCs in similarly massive 'old' ellipticals. Metallicity estimates from spectra of young GCs in NGC 1275 and NGC 7252 were hampered by relatively large uncertainties, since $\sim 500$ Myr old populations are dominated by A-type stars with intrinsically weak metallic features.

Observations of more evolved merger remnants were obviously necessary to make substantial progress on these issues. However, intermediate-age merger 
remnants (with ages of $1-5$ Gyr) are more difficult to identify than younger remnants since the main body of the former has had enough time to relax into a relatively symmetric configuration and the newly-formed stellar populations have had time to fade and redden [13]. We [14] recently conducted multi-slit spectroscopy of GC candidates in NGC 1316, an early-type galaxy that is an obvious merger remnant 15. We discovered the presence of $\sim 10 \mathrm{GCs}$ associated with NGC 1316 that have luminosities higher than that of $\omega$ Cen (up to $\sim 10$ times that, actually). Our measurements of $\mathrm{H} \alpha$ and the Ca II triplet in the spectra of the brightest GCs showed them to have solar metallicity (to within 0.15 dex) and to have an age of $3.0 \pm 0.5$ Gyr. These are obviously GCs that have formed from enriched gas during a major merger. This reinforces the view that luminous GCs formed during mergers do not necessarily have high low-mass cutoffs to their IMFs. Another important implication is that these GCs can actually survive dynamical disruption processes taking place during and after the merging process (see below).

\section{The Luminosity Function of GCs Formed in Mergers}

The luminosity functions (LFs) for GCs in young mergers (such as the Antennae or NGC 7252) are power laws with indices of $\alpha \sim-2$ [16]; [17]. This distribution contrasts markedly with the Gaussian profiles found for LFs of old GC systems such as that of the Milky Way or giant ellipticals [18]. However, various destruction mechanisms - such as 2-body evaporation, bulge and disk shocking, and stellar mass loss- should remove the less massive and/or more diffuse clusters as the galaxy ages. Hence, a system of GCs created during a merger may eventually yield a peaked distribution of clusters similar to what is seen for old GCs 19, 20]. Intermediate-age remnants, such as NGC 1316 [14 and NGC 3610 13, offer the possibility of actually observing this process in action. While the blue, metal-poor GCs in these galaxies should have an LF similar to that of old GCs in elliptical galaxies (peaking at $M_{V} \approx-7.2$ 21]), the LFs of the red, metal-rich clusters are expected to extend to both brighter and fainter luminosities.

This prediction has recently been verified for two intermediate-age merger remnants, providing important evidence that mergers can indeed create "red" GC subsystems that survive destruction. Goudfrooij et al. [20] used WFPC2 photometry of the GC system of the 3-Gyr-old merger remnant NGC 1316. By using appropriate color cuts in the color-magnitude diagram of NGC 1316's GC system, they showed that indeed, the LF for the blue GCs is approximately Gaussian centered on the expected turnover magnitude, while the LF for the red GCs is more nearly power-law shaped down to the completeness limit of the data (see Fig. 1 1a,b). Likewise, Whitmore et al. 22 performed deep WFPC2 photometry of the GC system of the candidate 4-Gyr-old merger remnant NGC 3610 [13], [23]. They found a very similar result, with the red GCs still showing a power-law LF shape (see Fig. 1 $1 \mathrm{c}, \mathrm{d}$ ). 

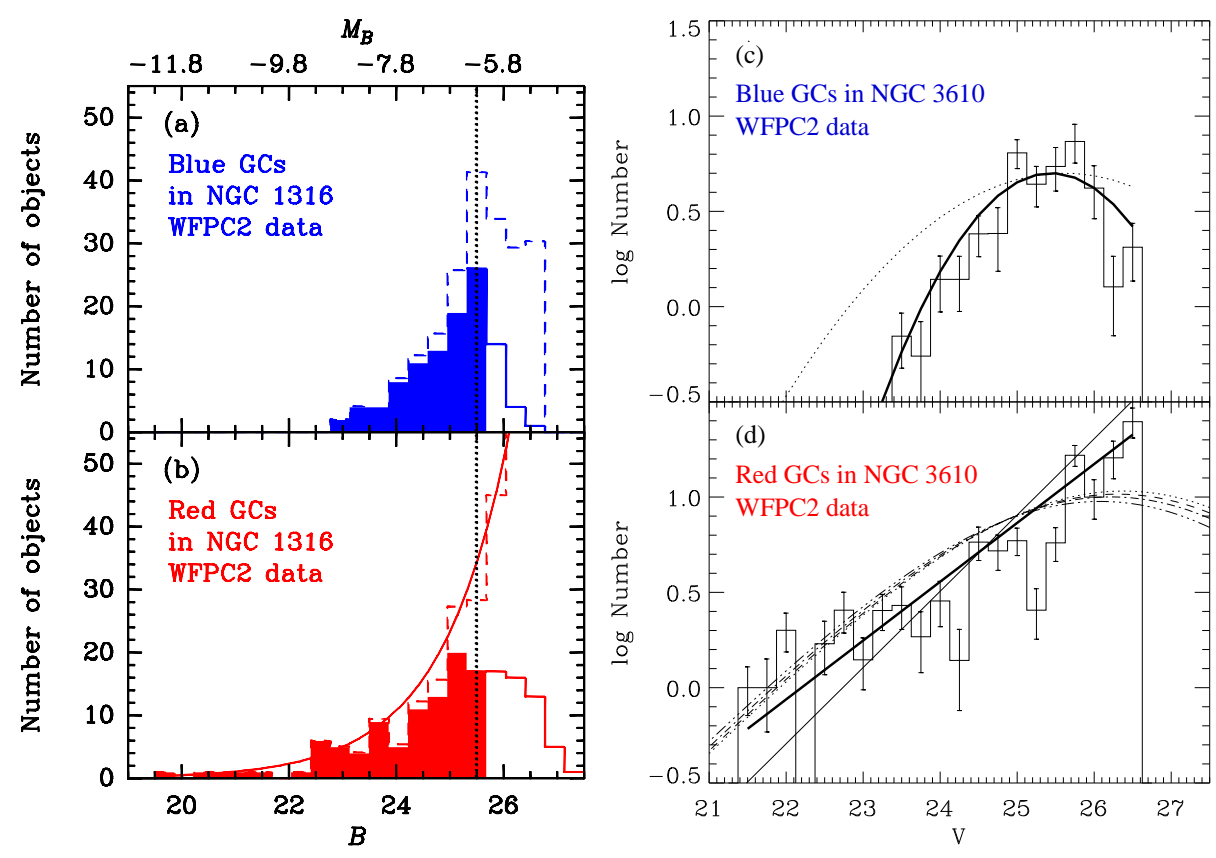

Fig. 1. Luminosity functions (LFs) of GC candidates in NGC 1316 and NGC 3610 using HST/WFPC2 data, taken from Goudfrooij et al. 14 and Whitmore et al. 22, respectively. The top left panel (a) shows the LF for the blue GC candidates in NGC 1316 (with $1.00 \leq B-I \leq 1.55$ ), panel (b) does so for the red $(1.75 \leq B-I \leq 2.50)$ GC candidates in NGC 1316, panel (c) does so for the blue GC candidates in NGC 3610, and panel (d) does so for the red GC candidates in NGC 3610. For panels (a) and (b), the top axis unit is absolute magnitude $M_{B}$ at the distance of NGC 1316. The histograms are filled for magnitudes below the 50 per cent completeness limit, and open beyond it. Dashed lines in panels (a) and (b) show the effect of completeness and background corrections, while the dotted line at $B=25.5$ depicts the expected LF peak for the old GCs in NGC 1316. The data in panels (c) and (d) are corrected for completeness. Solid (thick) curves in panels (b) and (d) are power-law fits to the completeness-corrected luminosity functions. Other curves in panels (c) and (d) are described in ref. 22].

An important next step for progress in this field will be finding evidence for a metamorphosis of the power-law-like LF of those red GCs into a Gaussian shape as seen in old GC systems. This metamorphosis is now predicted theoretically (19], esp. their Fig. 4). Quantitative estimates of the LF shape evolution can be made by combining GC disruption model predictions (of mass functions) with $M / L$ ratio predictions from stellar population models. This was done by Whitmore et al. 22] who showed that after 1.5 - 2 Gyr, the effect of the shift of the peak of the GC mass function toward smaller masses (due to disruption processes) to the LF is essentially compensated by the luminosity fading of GCs. The net prediction is that at a given metallicity, the peak of the LF remains nearly constant with time in the range $1.5-12$ Gyr. This effect is depicted 


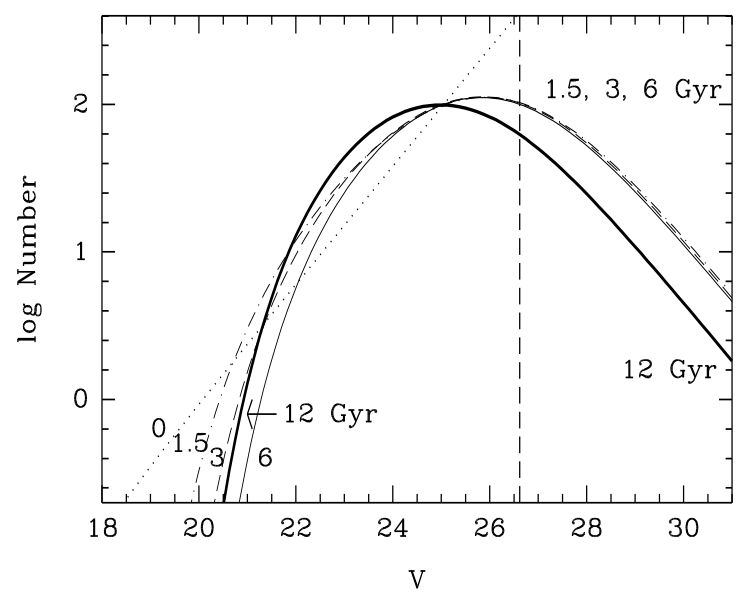

Fig. 2. Predicted evolution of LFs based on a combination of the Fall \& Zhang 19 cluster disruption models and the BC96 population synthesis models. Solar metallicity is assumed for the young and intermediate-age GCs while 0.02 solar is assumed for the 12 Gyr population. The LFs are normalized at $V=25$. Note that the fading predicted by the BC96 models almost exactly cancels out the increase in the mean mass predicted by the Fall and Zhang models, resulting in nearly identical distributions at the faint end for the 1.5, 3, and 6 Gyr models. Figure taken from ref. [22].

in Fig. 2. Note that the LF turnover for metal-rich, intermediate-age GCs is predicted to be $\sim 1$ mag fainter than that of the old, metal-poor GCs. This prediction will be tested in a forthcoming $H S T$ program utilizing the enormous discovery power of the Advanced Camera for Surveys (ACS).

\section{Specific Frequencies of GCs formed in Mergers}

One of the arguments often made against the concept that red GCs in giant ellipticals have been formed in gas-rich mergers is that the specific frequencies $\left(S_{N} \mathrm{~s}\right)$ of GCs in merger remnants are low relative to those in giant ellipticals. I would like to emphasize two things in this context. First, the number of GCs in the definition of $S_{N}$ was defined for "old" GC systems with a Gaussian LF, i.e., twice the number of GCs brighter than the turnover magnitude [24]. Hence, an appropriate calculation of $S_{N}$ for merger remnants requires a separate approach for 'blue' and 'red' GCs. The 'blue' GCs should be counted the normal way, but the luminosities of the 'red' GCs and of the host galaxy need to be faded to those appropriate for an age of (say) 10 Gyr. Also, the 'red' GCs need to be counted up to the turnover magnitude appropriate for a $10 \mathrm{Gyr}$ old population at the observed GC metallicity, calculated as described in Section 3. Specific frequencies for the intermediate-age merger remnants NGC 1316, NGC 1700 [23], and NGC 3610 calculated this way are listed in Table 1. A range of $S_{N}$ values 
is given for each galaxy, indicating the allowed range of the current fraction (by mass) of intermediate-age stars in the parent galaxy.

Table 1. Specific Frequencies $S_{N}$ for Intermediate-Age Merger Remnants

\begin{tabular}{ccc}
\hline Galaxy & \multicolumn{1}{c}{$S_{N}$} & Ref. of GC counts \\
\hline NGC 1316 & $2.1 \cdots 3.4$ & 20 \\
NGC 1700 & $1.6 \cdots 2.8$ & 23 \\
NGC 3610 & $1.7 \cdots 2.9$ & 22 \\
\hline
\end{tabular}

Second, the $S_{N}$ values for these three galaxies should be compared with those of 'normal' ellipticals in similar environment densities. NGC 1316 is in a poor group behind the Fornax cluster 25], 14]. NGC 3610 and NGC 1700 are in poor groups with 5 and 7 members, respectively [26]. Hence their $S_{N}$ values should be compared with the mean $S_{N}$ of ellipticals in poor groups, which is $2.6 \pm$ 0.5 [18]. A glance at Table 1 then shows that the specific frequencies of GCs in intermediate-age merger remnants are consistent with those of 'old' ellipticals.

\section{References}

1. K.M. Ashman, S.E. Zepf: ApJ 384, 50 (1992)

2. P. Côté, R.O. Marzke, M.J. West: ApJ 501, 554 (1998)

3. D.A. Forbes, J.P. Brodie, C.J. Grillmair: AJ 113, 1652 (1997)

4. B.C. Whitmore, F. Schweizer: AJ 109, 960 (1995)

5. S.E. Zepf, K.M. Ashman, J. English, et al.: AJ 118, 752 (1999)

6. J.P. Brodie, L.L. Schroder, J.P. Huchra, et al.: AJ 116, 691 (1998)

7. F. Schweizer, B.W. Miller, B.C. Whitmore, et al.: AJ 112, 1839 (1996)

8. M.N. Carlson, J.A. Holtzman, C.J. Grillmair, et al.: AJ 117, 1700 (1999)

9. B.C. Whitmore, F. Schweizer, C. Leitherer, et al.: AJ 106, 1354 (1993)

10. F. Schweizer, P. Seitzer: AJ 116, 2009 (1998)

11. J.S. Gallagher III, L.J. Smith: MNRAS 304, 540 (1999)

12. L.J. Smith, J.S. Gallagher III: MNRAS 326, 1027 (2001)

13. F. Schweizer, P. Seitzer: AJ 104, 1039 (1992)

14. P. Goudfrooij, J. Mack, M. Kissler-Patig, et al.: MNRAS 322, 643 (2001a)

15. F. Schweizer: ApJ 237, 303 (1980)

16. B.W. Miller, B.C. Whitmore, F. Schweizer, S.M. Fall: AJ 114, 2381 (1997)

17. B.C. Whitmore, Q. Zhang, C. Leitherer, et al.: AJ 118, 1551 (1999)

18. W.E. Harris: ARAA 29, 543 (1991)

19. S.M. Fall, Q. Zhang: ApJ 561, 751 (2001)

20. P. Goudfrooij, M.V. Alonso, C. Maraston, et al.: MNRAS 328, 237 (2001b)

21. B.C. Whitmore: in The Extragalactic Distance Scale, ed. by M. Livio, M. Donahue \& N. Panagia (STScI, Baltimore), p. 254

22. B.C. Whitmore, F. Schweizer, A. Kundu, B.W. Miller: AJ 124, 147 (2002)

23. B.C. Whitmore, B.W. Miller, F. Schweizer, et al.: AJ 114, 1797 (1997)

24. W.E. Harris, S. van den Bergh: AJ 86, 1627 (1981)

25. M.J. Drinkwater, M.D. Gregg, M. Colless: ApJ 548, L139 (2001)

26. A.M. Garcia: A\&AS 100, 47 (1993) 